\title{
Dissipative hydrodynamics coupled to chiral fields
}

\author{
J. Peralta-Ramos* and G. Krein ${ }^{\dagger}$ \\ Instituto de Física Teórica, Universidade Estadual Paulista, Rua Doutor Bento Teobaldo Ferraz, 271-Bloco II, \\ 01140-070 São Paulo, SP, Brazil
}

(Received 28 July 2011; published 7 October 2011)

\begin{abstract}
Using second-order dissipative hydrodynamics coupled self-consistently to the linear $\sigma$ model we study the $2+1$ dimensional evolution of the fireball created in $\mathrm{Au}+\mathrm{Au}$ relativistic collisions. We analyze the influence of the dynamics of the chiral fields on the charged-hadron elliptic flow $v_{2}$ and on the ratio $v_{4} /\left(v_{2}\right)^{2}$ for a temperature-independent as well as for a temperature-dependent viscosity-to-entropy ratio $\eta / s$ calculated from the linearized Boltzmann equation in the relaxation time approximation. We find that $v_{2}$ is not very sensitive to the coupling of chiral sources to the hydrodynamic evolution, but the temperature dependence of $\eta / s$ plays a much bigger role on this observable. On the other hand, the ratio $v_{4} /\left(v_{2}\right)^{2}$ turns out to be much more sensitive than $v_{2}$ to both the coupling of the chiral sources and the temperature dependence of $\eta / s$.
\end{abstract}

DOI: 10.1103/PhysRevC.84.044904

PACS number(s): 25.75.-q, 24.10.Nz, 24.85.+p

\section{INTRODUCTION}

One of the most surprising outcomes of the experiments conducted at the Relativistic Heavy Ion Collider (RHIC) is the discovery that high-energy collisions of heavy ions produce strongly interacting hadronic matter [(quarkgluon plasma (QGP)] that evolves as a low-viscosity fluid [1-6]. This came as a surprise because early expectations, motivated mainly by the asymptotic freedom property of quantum chromodynamics (QCD), were that RHIC would create a gaslike system of weakly interacting quarks and gluons.

The strongly interacting nature of the QGP is revealed through the description of measured momentum anisotropies via relativistic viscous hydrodynamics calculations. The momentum anisotropies are encoded in the Fourier moments $v_{2}$, $v_{3}, v_{4}, \ldots$ of the measured azimuthal distribution of particles, and are interpreted as being the translation to momentum space of the initial spatial eccentricity of noncentral collisions [7]. A weakly interacting, gaslike fluid would have no mechanism to induce such a translation into momentum anisotropies, but they can be produced if the particles of the fluid are strongly interacting.

In any attempt of describing experimental results of a heavy-ion collision via a viscous hydrodynamics model, crucial physics input reflecting the properties of the flowing hadronic matter must be supplemented, like the equation of state (EOS) and transport coefficients, as shear $(\eta)$ and bulk $(\zeta)$ viscosities. Although it is expected that for the strongly coupled QGP $\eta$ and $\zeta$ will depend strongly on temperature - see below-usually in hydrodynamic simulations temperature-independent values for these coefficients are assumed throughout the entire evolution. The impact of the temperature dependence of transport coefficients on momentum anisotropies that are obtained from hydrodynamic models was recently investigated in Refs. [8-14].

*jperalta@ift.unesp.br

${ }^{\dagger}$ gkrein@ift.unesp.br
These transport coefficients are, in principle, derivable from first-principles QCD calculations at finite temperature, but in practice, the calculations are extremely difficult at strong coupling. There is a long history of computations of the EOS via large-scale numerical simulations of QCD on a lattice and modern calculations are achieving the accuracy needed for reliable use in heavy-ion physics-for a recent overview on the subject, see Ref. [15]. On the other hand, lattice QCD calculations of transport coefficients are still in their infancy and only recently have results for shear and bulk viscosities been obtained [16-18]. Analytically, calculations have been performed within QCD for the shear and bulk viscosities $[19,20]$ at extremely high temperatures, where perturbation theory in the coupling constant can be used on account of asymptotic freedom. Reliable calculations for these quantities can also be done at very low temperatures, where matter consists essentially of a very dilute gas of pions. There is plenty of experimental information on elementary low-energy hadron-hadron interactions available that can be used to constrain detailed calculations that go beyond lowest order in chiral perturbation theory [21,22]. On the other hand, in the region of intermediate temperatures, $T \sim \Lambda_{\mathrm{QCD}}$, where one expects the deconfinement and chiral transitions to occur [23], perturbative expansions (in the coupling constant or chiral) are not applicable. There is lack of experimental information on the appropriate degrees of freedom to use and phenomenological approaches are an alternative, like the use of relativistic kinetic theory [24-30].

Assuming the validity of a relativistic viscous hydrodynamical description, experimental extraction of transport coefficients should be possible in principle from comparison with measured momentum anisotropy patterns. Moreover, as suggested in Ref. [31], such a comparison with experimental data would allow one to pinpoint the location of the QCD phase transition or of a crossover from hadronic to quark-gluon matter. In this context, in addition to the hydrodynamic degrees of freedom related to energy-momentum conservation, degrees of freedom associated with order parameters of broken continuous symmetries must be considered as well because they are all coupled to each other. Particularly important to the 
quest of determining possible signals of the chiral transition is the coupling of chiral fields to the fluid-dynamical modes. The present work is a first step toward the study of effects of such a coupling on momentum anisotropies within a viscous hydrodynamic description.

Specifically, in the present paper we study, in the context of second-order dissipative relativistic hydrodynamics, the influence of the long-wavelength modes of chiral fields on the expansion of the fireball created in $\mathrm{Au}+\mathrm{Au}$ collisions at $\sqrt{s_{N N}}=200 \mathrm{GeV}$. In particular, we aim at studying the effect of the coupled evolution of chiral degrees of freedom on the flow asymmetry characterized by $v_{2}$ and $v_{4} /\left(v_{2}\right)^{2}$, when finite viscosity is taken into account within a simple microscopic model for the chiral condensate.

In our model the flowing matter consists of a fluid of quarks and antiquarks in local thermal equilibrium that evolves according to a second-order dissipative hydrodynamic model [32-34] with temperature-dependent speed of sound and transport coefficients. Starting from a high-temperature state with approximately restored chiral symmetry, the system is evolved toward a state where the symmetry is spontaneously broken. The quark-antiquark fluid interacts locally with the chiral fields of the linear $\sigma$ model (LSM) [35]. We compare the results obtained for hadronic observables when the chiral fields are included or not as sources in the hydrodynamic equations.

Similar models for the dynamics of quarks coupled to chiral fields were considered before. Mishustin and Scavenius [36] constructed a relativistic mean-field ideal fluid dynamical model based on the LSM, while Abada and Birse [37] used the Vlasov equation to describe the quarks coupled to the LSM. Both studies focused on the evolution of the chiral fields rather than on the influence of their dynamics on hadronic observables, which is the main focus here. Son [38] and Pujol and Davesne [39] have developed modified hydrodynamic theories including additional (chiral) symmetry-breaking hydrodynamic variables, which were subsequently applied by Lallouet et al. [40] to the study of Bjorken flow in heavy-ion collisions. Later on, Paech et al. [41,42] coupled 3+1 ideal hydrodynamics to the LSM including fluctuations of the chiral fields and studied the behavior of the azimuthal momentum asymmetry at the nonzero impact parameter. More recently, Nahrgang and Bleicher [43] included phenomenological dissipation and noise terms in the equation of motion of the $\sigma$ field of the LSM coupled to ideal hydrodynamics. They focused on sigma fluctuations and found that at the first-order phase transition they lead to an increase in the intensity of sigma excitations. In a subsequent work, Nahrganget al. [44] employed the two-particle irreducible (2PI) effective action formalism to set up an approximation scheme to derive explicit formulas for the dissipation kernel and the noise correlation function within the LSM. While the approximations employed deserve careful scrutiny, the formulas derived within the mentioned approximation scheme show interesting features of dissipation and noise on the relaxation dynamics of the chiral dynamics and point to the importance of including reheating of the quark fluid by energy dissipation from the chiral fields, as shown in the very recent papers by Nahrgang, Leupold, and Bleicher [45] and Nahrgang, Bleicher, Leupold, and Mishustin [46]. The work of Plumari et al. [47] is more in the spirit of ours in the present paper, in that they have investigated the role of the chiral transition on $v_{2}$. Specifically, the authors have solved numerically the Boltzmann-Vlasov transport equations including both two-body collisions and the chiral phase transition as given by the Nambu-Jona-Lasinio model. For conditions prevailing in $\mathrm{Au}+\mathrm{Au}$ collisions at $\sqrt{s_{N N}}=200 \mathrm{GeV}$, the authors find a sizable suppression of $v_{2}$ because of the attractive nature of the chiral field dynamics. They also found that, if $\eta / s$ is kept fixed, $v_{2}$ does not depend on the details of the collisional and/or field dynamics and in particular it is not affected significantly by the chiral phase transition.

At this point it is appropriate to state the limited scope of our work. First of all, while we use a viscous hydromodel coupled to a workable chiral model, we do not intend to extract values of transport coefficients by comparing our results to $v_{2}$ data. Actually, extraction of transport coefficients by matching the output of hydrodynamic and/or kinetic simulations to data is not at all straightforward, as discussed, for example, in Refs. [9,48-50]. In particular, to extract $\eta / s$ from $v_{2}$ measurements, a possible route is to first constrain initial $T_{i}$ and final $T_{f}$ temperatures (that are used to run the coupled set of hydrochiral equations) by matching calculated spectra to data and, afterward, extract $\eta / s$ from $v_{2}$ data (see, e.g., Refs. [48,51]). In addition, although we use a semirealistic initial condition (we use a Glauber smooth initial condition), a fluctuating, lumpy initial condition leads to lower elliptic flow along with other interesting effects [52-54] that certainly have an impact on the extraction of transport coefficients.

The paper is organized as follows. In Sec. II we describe the coupling of the chiral fields to the $2+1$ hydrodynamic model. In Sec. III we describe the initial conditions and the freeze-out prescription used in the simulations. We then show and discuss our results in Sec. IV, and conclude in Sec. V. In Appendix A we briefly overview the LSM and the calculation of the speed of sound and of the shear viscosity from the linearized Boltzmann equation in the relaxation time approximation. In Appendix B we discuss the dependence of our results on the cut-off that we must impose on the value of $\eta / s$ due to the breakdown of viscous hydrodynamics when nonequilibrium effects start to dominate.

\section{COUPLED HYDROCHIRAL DYNAMICS}

In this section we describe the model of coupling chiral fields to hydrodynamical variables. The evolution of the chiral fields is described by the LSM [35] and hydrodynamics is described by dissipative hydrodynamic equations [32-34]. This hydrodynamic formalism goes beyond the well-known Israel-Stewart theory [55-57] in that it includes all secondorder terms in velocity gradients that can appear in the stress-energy tensor of a conformal fluid (see, e.g., Ref. [58]). This hydromodel was applied to model the expansion of the QGP in Refs. [48,51].

To set notation and make the paper self-contained, we start with the LSM model. The Lagrangian density of the model, given in terms of the quark $q=(u, d)$ and chiral $\phi_{a}=(\sigma, \vec{\pi})$ 
fields, is written as [we use the signature for the metric $g_{\mu \nu}=$ $(+,-,-,-)]$

$$
\mathcal{L}=\mathcal{L}_{q}+\mathcal{L}_{\phi}
$$

with

$$
\mathcal{L}_{q}=\bar{q}\left[i \gamma^{\mu} \partial_{\mu}-g\left(\sigma+\gamma_{5} \vec{\tau} \cdot \vec{\pi}\right)\right] q
$$

and

$$
\mathcal{L}_{\phi}=\frac{1}{2}\left(\partial_{\mu} \sigma \partial^{\mu} \sigma+\partial_{\mu} \vec{\pi} \cdot \partial^{\mu} \vec{\pi}\right)-U(\sigma, \vec{\pi}),
$$

where

$$
\begin{aligned}
U(\sigma, \vec{\pi}) & \equiv U(\phi)=\frac{\lambda^{2}}{4} \phi^{2}-h_{q} \sigma-U_{0} \\
& =\frac{\lambda^{2}}{4}\left(\sigma^{2}+\pi^{2}-v^{2}\right)^{2}-h_{q} \sigma-U_{0},
\end{aligned}
$$

is the potential which exhibits chiral symmetry breaking - with $\phi^{2}=\sigma^{2}+\pi^{2}$. At the level of approximation we work in the present paper, parameters are fixed as follows. The vacuum expectation values of the condensates are taken $\langle\sigma\rangle=f_{\pi}$ and $\langle\vec{\pi}\rangle=0$, with the pion decay constant $f_{\pi}=93 \mathrm{MeV}$. Also, the partially conserved axial-vector current relation yields $h_{q}=$ $f_{\pi} m_{\pi}^{2}$ with $m_{\pi}=138 \mathrm{MeV}$. This leads to $v^{2}=f_{\pi}^{2}-m_{\pi}^{2} / \lambda^{2}$. A mass $m_{\sigma}=\sqrt{2 \lambda^{2} f_{\pi}^{2}+m_{\pi}^{2}} \sim 600 \mathrm{MeV}$ is obtained if $\lambda^{2}=$ 20 . The constant $U_{0}$ is chosen such that the potential energy vanishes in the ground state.

Following Paech et al. [41,42], we split the energymomentum tensor as

$$
T^{\mu \nu}=T_{q}^{\mu \nu}+T_{\phi}^{\mu \nu}
$$

As mentioned previously, the quarks and antiquarks are assumed to constitute a heat bath in local thermal equilibrium and their dynamical evolution will be determined by viscous relativistic hydrodynamics, so that

$$
T_{q}^{\mu \nu}=(\epsilon+p) u^{\mu} u^{\nu}-p g^{\mu \nu}+\Pi^{\mu \nu},
$$

where $u_{\mu}$ is the four velocity normalized as $u_{\mu} u^{\mu}=1, \Pi^{\mu v}$ is the viscous shear tensor, and $\epsilon=\epsilon(\phi, T)$ and $p=p(\phi, T)$ are the energy density and pressure (by definition) at equilibrium at local temperature $T$ (their explicit expressions are given in Appendix A). The contribution from the chiral fields to the energy-momentum tensor is given by

$$
T_{\phi}^{\mu \nu}=\sum_{a=0}^{4} \frac{\partial\left\langle\mathcal{L}_{\phi}\right\rangle}{\partial\left(\partial_{\mu} \phi_{a}\right)} \partial^{\nu} \phi_{a}-g^{\mu \nu}\left\langle\mathcal{L}_{\phi}\right\rangle,
$$

where $\langle\cdots\rangle$ means local thermal average.

The hydrodynamic equations of motion are obtained from conservation of the energy-momentum tensor, $D_{\mu} T^{\mu \nu}=$ 0 , where $D_{\mu}$ is the geometric covariant derivative. We employ Milne coordinates [appropriate for a $(2+1)$ flow dynamics] defined by proper time $\tau=\sqrt{t^{2}-z^{2}}$ and rapidity $\psi=\operatorname{arctanh}(z / t)$. The hydrodynamic velocity is $u=\left(u^{\tau}, u^{x}, u^{y}, 0\right)$ and the metric tensor reads $g_{\mu \nu}=$ $\left(-1,1,1, \tau^{2}\right)$. In the transverse plane we use Cartesian coordinates $x^{i}=(x, y)$, so that the only nonvanishing Christoffel symbols are $\Gamma_{\psi \psi}^{\tau}=\tau$ and $\Gamma_{\tau \psi}^{\psi}=1 / \tau$. Because we assume boost invariance, all quantities are independent of rapidity. The conservation equations then read

$$
D \epsilon=-(\epsilon+p) \nabla_{\mu} u^{\mu}+\Pi^{\mu v} \sigma_{\mu \nu}+g\left(\rho_{s} D \sigma+\vec{\rho}_{p s} \cdot D \vec{\pi}\right),
$$

$$
\begin{aligned}
(\epsilon+ & p) D u^{i} \\
= & c_{s}^{2}\left(g^{i j} \partial_{j} \epsilon-u^{i} u^{\alpha} \partial_{\alpha} \epsilon\right)-\Delta_{\alpha}^{i} D_{\beta} \Pi^{\alpha \beta} \\
& +g\left(\rho_{s} \nabla^{i} \sigma+\vec{\rho}_{p s} \cdot \nabla^{i} \vec{\pi}\right)
\end{aligned}
$$

where $\Delta^{\mu \nu}=g^{\mu \nu}-u^{\mu} u^{\nu}$ is a projector (orthogonal to the fluid velocity), $c_{s}$ is the speed of sound, and $\rho_{s}=\langle\bar{q} q\rangle$ and $\vec{\rho}_{p s}=\left\langle\bar{q} \gamma_{5} \vec{\tau} q\right\rangle$ are the local thermal averages of the scalar and pseudovector chiral densities (their explicit expressions are given in Appendix A). It is seen that the chiral densities act as sources for the evolution of the hydrodynamic variables $\epsilon$ and $u^{\mu}$. Here, $D=u_{\mu} D^{\mu}$ is the comoving time derivative, $\nabla_{\mu}=\Delta_{\mu \alpha} D^{\alpha}$ is the spatial gradient, $\sigma_{\mu \nu}$ is the shear tensor:

$$
\sigma^{\mu \nu}=\nabla^{\langle\mu} u^{\nu\rangle}
$$

The angular braces around Lorentz indices denotes the spatial, symmetric, and traceless part of a tensor, that is, if $A^{\mu \nu}$ is a tensor then $A^{\langle\mu \nu\rangle}$ means

$$
A^{\langle\mu \nu\rangle}=\frac{1}{2}\left(\Delta^{\mu \alpha} \Delta^{\gamma \nu}+\Delta^{\mu \gamma} \Delta^{\alpha \nu}-\frac{2}{3} \Delta^{\mu \nu} \Delta^{\alpha \gamma}\right) A_{\alpha \gamma} .
$$

The evolution of the shear tensor depends only indirectly on the chiral fields and it is given by

$$
\begin{aligned}
\partial_{\tau} \Pi^{i \alpha}= & -\frac{4}{3 u^{\tau}} \Pi^{i \alpha} \nabla_{\mu} u^{\mu}-\frac{1}{\tau_{\pi} u^{\tau}} \Pi^{i \alpha}+\frac{\eta}{\tau_{\pi} u^{\tau}} \sigma^{i \alpha} \\
& -\frac{\lambda_{1}}{2 \tau_{\pi} \eta^{2} u^{\tau}} \Pi_{\mu}^{<i} \Pi^{\alpha>\mu}-\frac{u^{i} \Pi_{\mu}^{\alpha}+u^{\alpha} \Pi_{\mu}^{i}}{u^{\tau}} D u^{\mu} \\
& -\frac{u^{j}}{u^{\tau}} \partial_{j} \Pi^{i \alpha},
\end{aligned}
$$

where $\eta$ is the shear viscosity and $\left(\tau_{\pi}, \lambda_{1}\right)$ are second-order transport coefficients. We note that Israel-Stewart formalism is recovered from these equations when $\lambda_{1}=0$.

In the Milne coordinates, the classical equations of motion for the chiral fields evolving in the background of the quark fluid read

$$
\begin{aligned}
& \left(\partial_{\tau}^{2}+\frac{1}{\tau} \partial_{\tau}-\partial_{i}^{2}\right) \sigma+\frac{\delta U}{\delta \sigma}=-g \rho_{s}, \\
& \left(\partial_{\tau}^{2}+\frac{1}{\tau} \partial_{\tau}-\partial_{i}^{2}\right) \vec{\pi}+\frac{\delta U}{\delta \vec{\pi}}=-g \vec{\rho}_{p s},
\end{aligned}
$$

where $\rho_{s}$ and $\vec{\rho}_{p s}$ are the scalar and pseudoscalar chiral condensates defined above.

In this way, the evolution of the chiral fields affects the evolution of the quark fluid through the sources in the energymomentum conservation equations; in turn, the quark fluid affects the evolution of the chiral fields through the densities $\rho_{s}$ and $\vec{\rho}_{p s}$.

\section{SOLVING THE EQUATIONS}

We solve the hydrodynamic equations given in Eqs. (8) and (9) using the publicly available code of Luzum and 
Romatschke [48]. We need to supply the locally temperaturedependent transport coefficients $\eta, \tau_{\pi}$, and $\lambda_{1}$, and speed of sound $c_{s}$. In addition, we need to specify initial conditions for the energy density $\epsilon$, velocities $u_{1}$ and $u_{2}$, and the components of the shear tensor. To solve the equations of motion for the chiral fields, Eqs. (13) and (14), we use a simple finite-difference scheme both in the proper-time and space variables. Here, also, one needs to provide initial conditions for the fields and their derivatives.

In all calculations performed in the present paper, we use a $13-\mathrm{fm} \times 13-\mathrm{fm}$ transverse plane. In the next subsections we discuss the values used for the input constants and initial conditions.

\section{A. Transport coefficients and the speed of sound}

As a prototypical example of a relativistic fluid, we will use $\tau_{\pi}=2(2-\ln 2) \eta / s T$ and $\lambda_{1}=\eta / 2 \pi T$, that correspond-in a gradient expansion at second order-to a supersymmetric Super-Yang-Mills plasma [32-34]. However, as mentioned in Sec. I, we will not use $c_{s}^{2}=1 / 3$ and $\eta / s=1 / 4 \pi$ appropriate to the Super-Yang-Mills plasma. The temperature dependence of the speed of sound is obtained within the LSM and that of $\eta$ is obtained using the linearized Boltzmann equation in the relaxation time approximation within the chiral model of Ref. [28]. We discuss these in Appendix A.

We note that the value that we use for the relaxation time $\tau_{\pi}=2(2-\ln 2) \eta / s T$ is not the exact value of the Super-YangMills plasma $[59,60]$. Rather, it is the value obtained through a second-order gradient expansion, that is equivalent to a Taylor expansion of the retarded Green's function that linearly relates a dissipative current and a thermodynamical force. The expression for $\tau_{\pi}$ derived in this way is in general different from the one obtained directly from the first pole of the retarded Green's function. Moreover, as shown in Refs. [59,60], the true dynamics of the long-wavelength and low-frequency modes of strongly coupled theories is not generally described by relaxation-type equations (i.e., the evolution equation for the shear tensor includes terms with second time derivatives of the thermodynamic forces;- see also Ref. [61]).

In this connection, it is important to remark that it was shown in previous hydrodynamic simulations (without chiral fields) [48,51] that, for values of $\eta / s \lesssim 0.2$, the results for the hadronic observables do not depend strongly on the choices for the second-order transport coefficients, provided that $\lambda_{1} \neq 0$. The influence of terms with second time derivatives of the thermodynamic forces in the evolution of the shear tensor of the QGP is still an open issue that surely deserves further investigation. In this work we will neglect such terms and use instead the relaxation-type equation given in Eq. (12), that is obtained from a second-order gradient expansion.

\section{B. Initial conditions}

For the initial transverse velocity and shear tensor we use $u^{x}=u^{y}=0$, which implies vanishing initial vorticity, and $\left(\Pi^{x x}, \Pi^{x y}, \Pi^{y y}\right)=0$. The initialization time is set to $\tau_{0}=1$ $\mathrm{fm} / \mathrm{c}$. It was shown before that the evolution of the shear tensor $\Pi^{\mu v}$ is quite insensitive to the initialization values, the difference being appreciable only at very early times (see, e.g., Ref. [50]). We have verified that the elliptic flow shows very little sensitivity to the initialization of the shear tensor as well. The initial temperature at the center of the fireball is set to $T_{i}=333 \mathrm{MeV}$. This value for $T_{i}$ was used in previous hydrodynamic simulations and leads to kaon multiplicity and $\left\langle p_{T}\right\rangle$ that are in good agreement with RHIC data $[48,51]$.

The initial energy density profile is calculated using Glauber's model, in which for a given impact parameter $b$ we have

$$
\epsilon\left(\tau_{0}, x, y, b\right)=C \sigma T_{A}(x+b / 2, y) T_{A}(x-b / 2, y),
$$

where $C$ is a constant chosen such that $\epsilon\left(\tau_{0}, 0,0,0\right)$ corresponds to a given initialization temperature $T_{0}$ via the EOS, the cross section $\sigma$ is taken to be $\sigma=40 \mathrm{mb}$, and $T_{A}$ is the nuclear thickness function given by

$$
T_{A}(x, y)=\int_{-\infty}^{\infty} \delta_{A}(x, y, z) d z
$$

with $\delta_{A}$ the density distribution for the gold nucleus, taken to be of a Woods-Saxon form,

$$
\delta_{A}(x, y, z)=\frac{\delta_{0}}{1+\exp \left[\left(|\vec{x}|-R_{0}\right) / \chi\right]},
$$

where $\vec{x}=(x, y, z), R_{0}=6.4 \mathrm{fm}$, and $\chi=0.54 \mathrm{fm}$. The parameter $\delta_{0}$ is chosen such that $\int d^{3} \mathbf{x} \delta_{A}(\mathbf{x})=197$, as appropriate for Au nuclei.

As a reasonable ansatz for the initial condition of the chiral fields we use

$$
\vec{\pi}\left(\tau_{0}, \vec{r}\right)=0 \quad \text { and } \quad \sigma\left(\tau_{0}, \vec{r}\right)=f_{\pi}\left[1-e^{-\left(r / r_{0}\right)^{2}}\right],
$$

with $r^{2}=x^{2}+y^{2}$ and $r_{0}=9 \mathrm{fm}$. In this way, the chiral condensate nearly vanishes at the center where the temperature of the fluid is larger and interpolates to $f_{\pi}$ where the temperature is lower.

\section{Freeze-out scheme}

In our simulations we use the isothermal Cooper-Frye freeze-out prescription [62] in which the conversion from hydrodynamic to particle degrees of freedom takes place in a three-dimensional hypersurface. The spectrum for a single on-shell particle with momentum $p^{\mu}=(E, \vec{p})$ and degeneracy $d$ is

$$
E \frac{d N}{d^{3} p}=\frac{d}{(2 \pi)^{3}} \int p_{\mu} d \Sigma^{\mu} f\left(x^{\mu}, p^{\mu}\right),
$$

where $d \Sigma^{\mu}$ is the normal vector on the hypersurface. The nonequilibrium distribution function $f$ is given by Grad's quadratic ansatz [63]:

$$
\begin{aligned}
f\left(x^{\mu}, p^{\mu}\right)= & f_{0}\left(x^{\mu}, p^{\mu}\right) \\
& +f_{0}\left(x^{\mu}, p^{\mu}\right)\left[1 \mp f_{0}\left(x^{\mu}, p^{\mu}\right)\right] \frac{p_{\mu} p_{\nu} \Pi^{\mu \nu}}{2 T^{2}(p+\epsilon)} \\
\simeq & {\left[1+\frac{p_{\mu} p_{\nu} \Pi^{\mu \nu}}{2 T^{2}(p+\epsilon)}\right] \exp \left(\frac{-p_{\mu} u^{\mu}}{T}\right), }
\end{aligned}
$$


where $f_{0}$ is the Fermi-Dirac distribution. The approximation in the third line holds when $p \gg T$, and it is used in our simulations. The systematic error of this approximation is very small at low transverse momentum $p_{T} \lesssim 2.5 \mathrm{GeV}$, so we do not expect our results to have a significant error coming from this approximation (see Ref. [48]).

We calculate the spectra for particle resonances with masses up to $2 \mathrm{GeV}$ and then determine the spectra of stable particles including feed-down contributions. For this last step we use the AZHYDRO package [64-66]. In the present paper we will focus on the elliptic flow coefficient $v_{2}$ and also on $v_{4}$ at central rapidity, which are related to the particle spectra (including feed-down contributions) by

$$
E \frac{d N}{d^{3} \vec{p}}=v_{0}\left(p_{T}, b\right)\left[1+\sum_{n=1}^{\infty} 2 v_{n}\left(p_{T}, b\right) \cos (n \varphi)\right],
$$

with $\varphi=\arctan \left(p_{y} / p_{x}\right)$ and $p_{T}=\left(p_{x}^{2}+p_{y}^{2}\right)^{1 / 2}$. For the freeze-out temperature we take $T_{f}=130 \mathrm{MeV}$. This value for $T_{f}$ is slightly smaller than the one usually employed in viscous hydrodynamic simulations of $\mathrm{Au}+\mathrm{Au}$ collisions of $T_{f} \sim 140 \mathrm{MeV}$, but allows us to study a broader range of temperatures near our value of $T_{c}$ to determine the influence of chiral fields dynamics on hadronic observables.

\section{RESULTS}

We now go over to discuss our results. We start with a very important input to the hydroequations, the square of the speed of sound $c_{s}^{2}=d p / d \epsilon$. In Fig. 1 we show $c_{s}^{2}$ as predicted by the LSM-its explicit expression is given in Appendix A-for three values of the coupling constant, namely $g=3.0,3.2,3.4$, which correspond to a smooth crossover. The reason to consider these values for $g$ is that recent works have shown that the crossover phase transition without a thermodynamic region where the sound velocity drops to zero leads to a faster time development of the system and helps to reproduce RHIC data with hydrodynamic

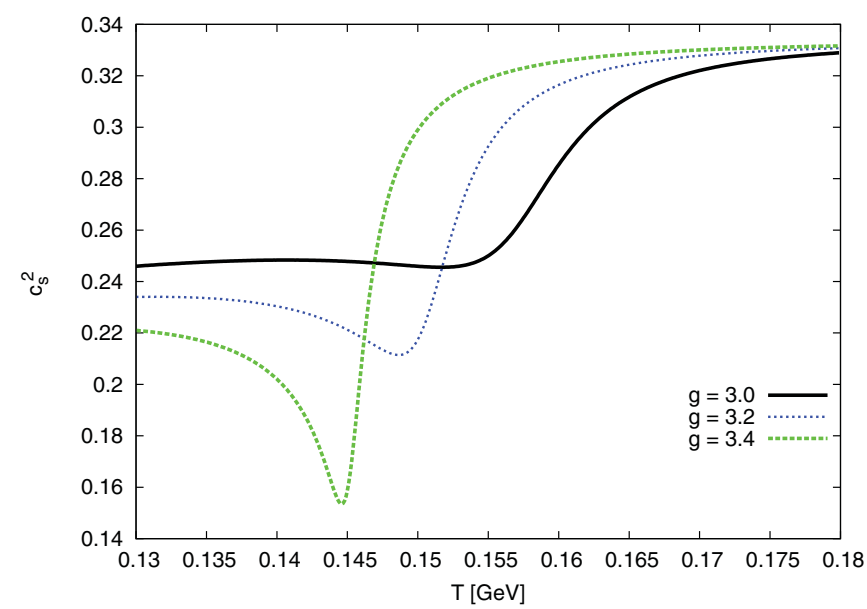

FIG. 1. (Color online) Square of the sound speed $c_{s}^{2}$ of the LSM as a function of temperature, for three values of the chiral coupling: $g=3,3.2,3.4$. simulations [67-69]. Moreover, lattice QCD calculations also favor a smooth crossover with a relatively soft dip in $c_{s}^{2}$ over a first-order phase transition (see, e.g., Refs. [70-74]). In our case, the first-order transition is obtained when $g=3.8$. As can be seen from Fig. 1, lowering the value of $g$ leads to a softening of the crossover and a rise in the critical temperature $T_{c}$. For large temperatures the conformal limit $c_{s}^{2}=1 / 3$ is reached, whereas for low values $c_{s}^{2}$ goes to zero. For $g \sim 3.2$ the behavior of $c_{s}^{2}$ with temperature is in qualitative agreement with that obtained in lattice QCD calculations [70-74] and with that favored by hydrodynamic simulations based on different interpolation schemes used to join lattice QCD and hadron resonance gas equations of state (see, e.g., Refs. [67-69]). For $g>3.4$ or $g<3$, the $\operatorname{dip}$ in $c_{s}^{2}$ at $T_{c}$ becomes very sharp or fades away, respectively, resulting in a temperature dependence that is in disagreement with the one obtained in lattice QCD calculations.

The critical temperature for these values of the LSM parameters is $T_{c} \sim 150 \mathrm{MeV}$, which is smaller than the value $\sim 170 \mathrm{MeV}$ obtained from lattice QCD simulations [70-74]. Within the LSM it is not possible to obtain much larger values for $T_{c}$ without going to very low values of $g$. These lower values of $g$ correspond to a very soft crossover with an almost inappreciable drop in $c_{s}^{2}$ that is not in agreement with the results the lattice QCD. The relatively low value for $T_{c}$ used here does not constitute a serious limitation to our aim of obtaining a qualitative understanding of the impact of the evolution of the chiral fields on hadronic observables, because we expect the trends obtained in this paper to hold in more realistic scenarios.

Another input to the hydrodynamic simulation is the ratio $\eta / s$ as a function of temperature, which we calculate in the LSM from the linearized Boltzmann equation in the relaxation time approximation (details are given in Appendix A). Figure 2 shows $\eta / s$ as a function of temperature in a region close to $T_{c}$, for $g=3,3.2,3.4$. It is seen that $\eta / s$ decreases significantly when approaching $T_{c}$ from below, and remains almost constant and rather small at $T>T_{c}$. This behavior is qualitatively similar to that found in other approaches such as the Boltzmann-hydrodynamics hybrid approach [75], the NJL model [28], and chiral perturbation theory [76], although, as

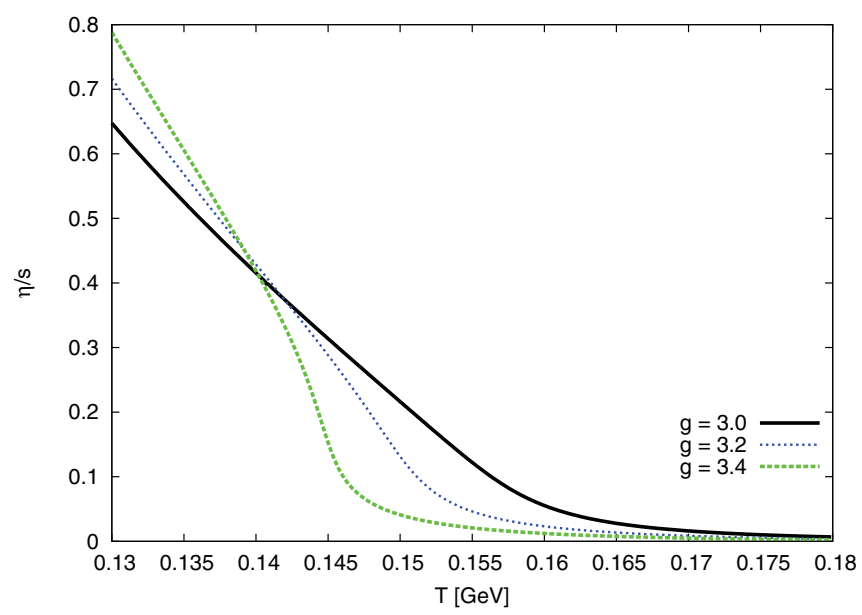

FIG. 2. (Color online) $\eta / s$ of the LSM as a function of temperature, for three values of the chiral coupling: $g=3,3.2,3.4$. 
already indicated, the value of $T_{c}$ obtained here is smaller. It is also seen that when the value of $g$ increases the variation of $\eta / s$ with temperature is more abrupt, decreasing faster at $T<T_{c}$ and thus remaining small for a larger range of temperatures.

At this stage it is appropriate to note an important point concerning the rise of $\eta / s$ with decreasing temperature in the low-temperature stage. By construction, any hydrodynamic description of matter necessarily breaks down when the viscosity is large enough that nonequilibrium effects are as important, or even more important than the equilibrium ones. For example, in the context of Grad's quadratic ansatz that is usually employed at freeze-out, this occurs when the nonequilibrium correction to the distribution function is comparable - or exceeds - the equilibrium one. In the context of relativistic heavy-ion collisions numerous hydrodynamic simulations (usually with temperature-independent $\eta / s$ ) have shown that large enough means $\eta / s \sim 0.3-0.5[10,13,50,75$, $77-80]$. For larger values of $\eta / s$ it is not possible to match the predictions of hydrodynamic models to RHIC data. Moreover, it was shown by direct comparison to results obtained with the Boltzmann equation that for matter created at RHIC, viscous hydrodynamics fails to reproduce the results of the Boltzmann equation if $\eta / s \gtrsim 0.2$ [81]. Beyond this value, an appropriate treatment of the dynamics of this matter should switch smoothly from a viscous hydrodynamic description to a kinetic one, in which the issue of breakdown from large viscosity (i.e., dissipation) does not arise. For recent developments and applications of this hybrid description see Refs. [10,75].

Because the model used here is purely hydrodynamic, a cutoff for the value of $\eta / s$ must be imposed to avoid the breakdown of the fluid description. The value of $\eta / s$ at which it is sensible to impose this cut-off is constrained by the comparison of results obtained from hydrodynamic and kinetic simulations to data. For this reason, in our simulations we set $\eta / s \leqslant 0.4$, which corresponds to $T \sim 140 \mathrm{MeV}$ for the three values of the chiral coupling, namely $g=3,3.2,3.4$, the values we consider in the present paper. We emphasize that in all of our simulations with $g=3,3.2,3.4$ the average value of $\eta / s$ throughout the hydrodynamic evolution is $\sim 0.11$, and thus it remains well below the value at which viscous hydrodynamics breaks down $(\eta / s \sim 0.3-0.5)$.

In Appendix B we analyze the dependence of our results on the choice of different cut-off values for $\eta / s$. We find that, although the values of $v_{2}$ and $v_{4} /\left(v_{2}\right)^{2}$ do change, the differences between the cases including or not the chiral fields as sources remain essentially the same, and thus our main conclusions are not sensitive to the value imposed for the cut-off.

Knowing $c_{s}^{2}$ and $\eta / s$ as functions of the temperature, we can now go over to discuss the momentum anisotropies obtained from the simulations. Figure 3 shows the charged-hadron elliptic flow $v_{2}$ calculated with either the temperature-dependent $\eta / s$ of the LSM or a temperature-independent $\eta / s=0.11$, which corresponds to the average of $\eta / s$ throughout the hydrodynamic evolution over the temperature interval from $T_{i}$ to $T_{f}$. In both cases, we set $g=3.2$ and compare the results obtained by taking or not taking into account the source terms in the hydrodynamic equations.

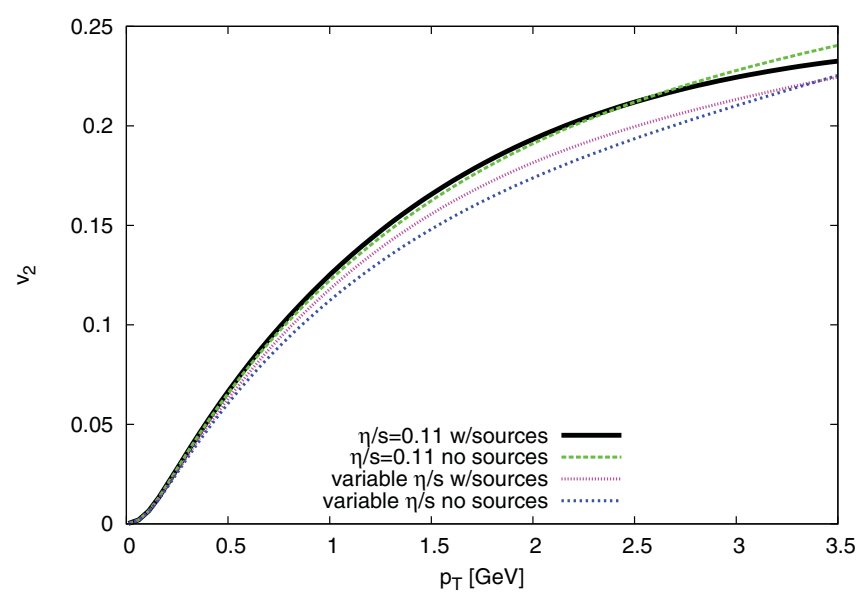

FIG. 3. (Color online) Elliptic flow of charged hadrons calculated taking or not taking into account the source terms in the hydrodynamic equations, for either a temperature-dependent or a temperatureindependent $\eta / s$ which is the averaged value throughout the evolution of the fireball. The value of the coupling constant is $g=3.2$.

It is seen from Fig. 3 that both for a temperature-dependent and a temperature-independent $\eta / s$, the elliptic flow does not depend strongly on the chiral sources. For a temperatureindependent $\eta / s, v_{2}$ turns out to be practically independent of the dynamics of the chiral sources for $p_{T}<3 \mathrm{GeV}$. In contrast, for the temperature-dependent $\eta / s$ there are small, but visible differences in the elliptic flow calculated with or without the chiral sources. Specifically, $v_{2}$ is slightly larger and reaches a maximum at $p_{T} \sim 2 \mathrm{GeV}$ when the chiral fields are taken into account as sources for the hydrodynamic variables.

Comparing the behavior of $v_{2}$ for temperature-independent or temperature-dependent $\eta / s$, it is seen that a temperaturedependent $\eta / s$ leads to a smaller elliptic flow. This fact was already seen in the studies of Refs. $[8,9,11]$ by performing hydrodynamic simulations (not coupled to chiral fields) with different parametrizations for temperature-dependent $\eta / s$ (usually a step-function dependence or some slight variation), and in Ref. [75] from a Boltzmann-hydrodynamics hybrid approach. See also Refs. [10,12] for related studies. In particular, it was found in Ref. [8] that $v_{2}$ at $\mathrm{Au}+\mathrm{Au}$ collisions at the largest RHIC energy is dominated by the shear viscosity in the hadronic phase and largely insensitive to the viscosity of the QGP. A similar conclusion was reached in Refs. [9,11]. It is interesting to note that, according to recent viscous hydrodynamic simulations with temperature dependent $\eta / s$, at LHC energies the situation is exactly the opposite (i.e., the elliptic flow becomes sensitive to the QGP viscosity and insensitive to the hadronic viscosity [8,9]). As noted in Ref. [9], the fact that $v_{2}$ depends rather weakly on the temperature dependence of $\eta / s$ poses serious challenges to the precise extraction of this ratio from this hadronic observable. For this reason, and also because of the uncertainty in the initial conditions in heavy-ion collisions at RHIC and LHC, it has now become important to calculate higher-order Fourier moments $v_{3}, \ldots$ in viscous hydrodynamic simulations, which may provide further crucial constraints on $\eta / s$ and on 
models used to calculate QGP initial conditions (see, e.g., Refs. [75,82-87]).

Returning to our results in Fig. 3, it seems clear the difficulties in extracting (an average value of) $\eta / s$ from data on $v_{2}$. The results show that uncertainties associated with the dependence of $\eta / s$ on temperature lead to appreciable changes in the curve of $v_{2}$ versus $p_{T}$. Such an uncertainty should be added to the theoretical uncertainty that comes, for example, from the initial conditions (e.g., using color glass condensate or Glauber initial conditions), the freeze-out process (as issues concerning Grad's quadratic ansatz), as well as from other sources, that according to recent studies add up to an overall uncertainty which can be roughly estimated in 0.1 [48,51].

As mentioned in Sec. I, the fact that $v_{2}$ depends somewhat on the evolution of the chiral fields in the case of a temperaturedependent $\eta / s$ was already seen in the study by Plumari et al. [47] within Boltzmann-Vlasov simulations on the NJL model. Our results show that this also happens in a purely hydrodynamic simulation as well.

Because our approach is phenomenological, it is important to determine the sensitivity of our conclusions regarding the influence of the chiral fields on $v_{2}$ to the value of the coupling constant $g$. Figure 4 shows the elliptic flow of charged hadrons calculated taking or not taking into account the source terms in the hydrodynamic equations, for $g=3,3.2,3.4$ and a temperature-dependent $\eta / s$. It is seen that when the value of $g$ increases, $v_{2}$ decreases. This is because of the fact that for larger values of $g, c_{s}^{2}$ near the transition region becomes smaller which means that pressure gradients are converted into flow less efficiently, thus resulting in lower values of $v_{2}$. The difference in the values of $v_{2}$ calculated including or not the chiral fields as sources is seen to become smaller with increasing $g$, which supports the conclusion reached earlier that the influence of the chiral fields on $v_{2}$ is rather small.

Another interesting observable to analyze is $v_{4} /\left(v_{2}\right)^{2}$, which was only recently theoretically investigated. See, in particular, Refs. [88,89] for a detailed account of the physics (and caveats; see below) involved in this observable within

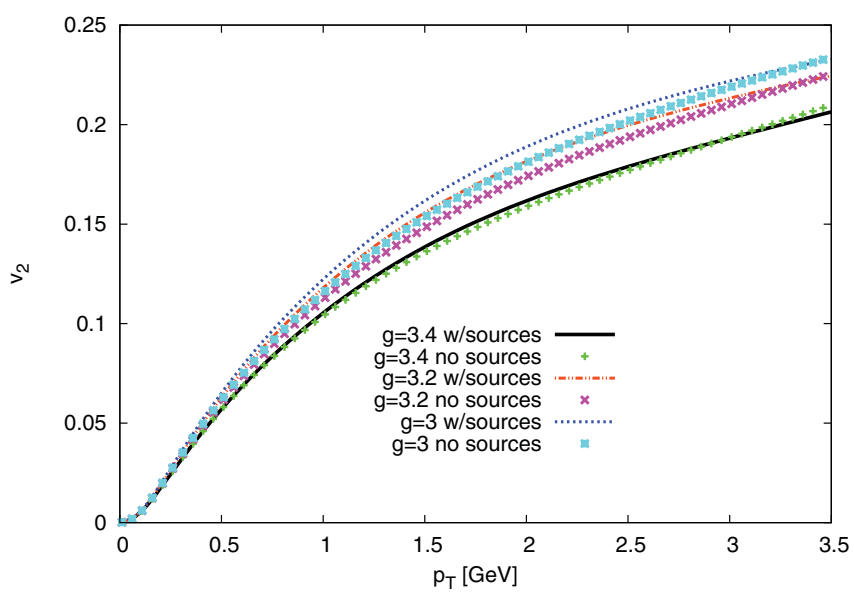

FIG. 4. (Color online) Elliptic flow of charged hadrons calculated taking or not taking into account the source terms in the hydrodynamic equations, for $g=3,3.2,3.4$ and a temperature-dependent $\eta / s$.

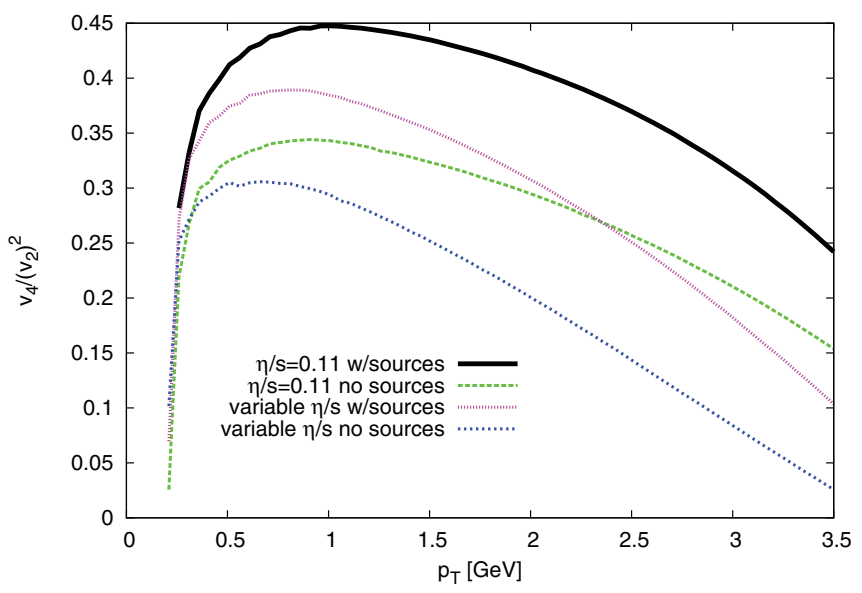

FIG. 5. (Color online) $v_{4} /\left(v_{2}\right)^{2}$ for charged hadrons calculated taking or not taking into account the chiral fields as sources for the hydrodynamic equations, for either a temperature-dependent or a temperature-independent $\eta / s$. The value of the coupling constant is $g=3.2$.

dissipative hydrodynamics. See also Ref. [47] for a calculation of $v_{4} /\left(v_{2}\right)^{2}$ within Boltzmann-Vlasov formalism. Figure 5 shows $v_{4} /\left(v_{2}\right)^{2}$ for the same cases as in Fig. 3. In contrast to what happens with $v_{2}$, it is seen that $v_{4} /\left(v_{2}\right)^{2}$ is significantly affected by the dynamics of the chiral fields even at low transverse momentum and for a temperature-independent $\eta / s$. In particular, the values of $v_{4} /\left(v_{2}\right)^{2}$ are larger when the chiral fields are included as sources in the hydrodynamic equations, and the behavior with $p_{T}$ is somewhat different too. It is also seen that a temperature-independent $\eta / s$ leads to larger $v_{4} /\left(v_{2}\right)^{2}$.

At this point, a comment on the possible extraction of $\eta / s$ from $v_{4} /\left(v_{2}\right)^{2}$ data by matching to hydrodynamic simulations is in order. It is known that the dependence of $v_{4} /\left(v_{2}\right)^{2}$ on transvserse momentum that is obtained from viscous hydrodynamics does not agree with the almost constant value of $v_{4} /\left(v_{2}\right)^{2} \sim 1$ measured at RHIC (see Refs. [88,89]). Ideal hydrodynamics predicts $v_{4} /\left(v_{2}\right)^{2}=0.5$, but quite surprisingly viscous hydrodynamics yields a strongly dependent ratio, as shown, for example, in Fig. 5. A possible explanation for this discrepancy was put forward by Luzum and Ollitrault $[88,89]$, who realized that Grad's quadratic ansatz for the nonequilibrium correction $\delta f$ to the particle distribution function may not be valid for the freeze-out process in heavy-ion collisions. See also Refs. [90-93] for recent work on the reliability of Grad's ansatz. By performing viscous hydrodynamic simulations with different dependencies of $\delta f$ on $p_{T}$, the authors of Refs. [88,89] have found that RHIC data on $v_{4} /\left(v_{2}\right)^{2}$ favors a momentum dependence between linear and quadratic. In spite of this, we have chosen to show results on $v_{4} /\left(v_{2}\right)^{2}$ to emphasize that, although the values for this observable are very different from those measured at RHIC (because we are using Grad's quadratic ansatz at freeze-out), they are much more sensitive than $v_{2}$ to the evolution of the chiral fields. We expect this feature of $v_{4} /\left(v_{2}\right)^{2}$ to hold when other forms for $\delta f$ are used in the simulations, although further work is needed to confirm this expectation. 


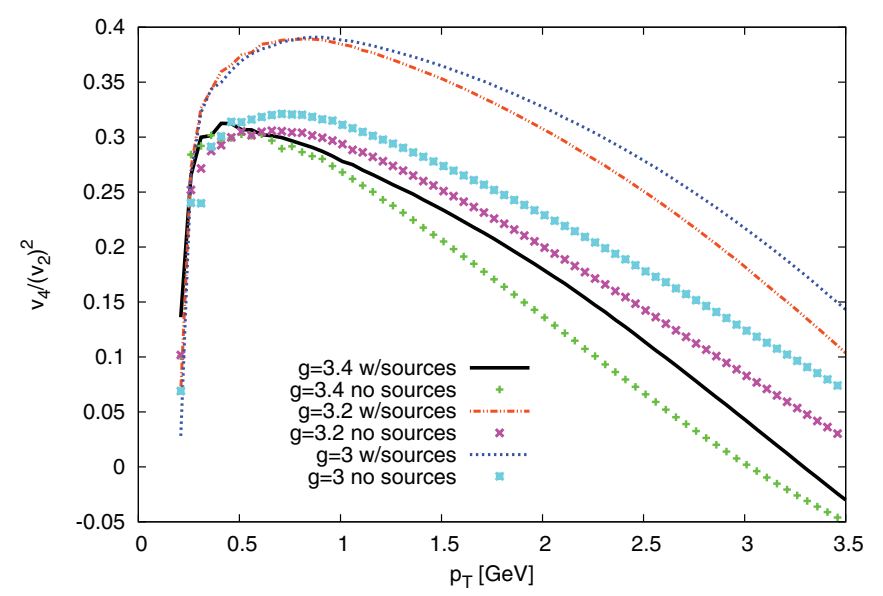

FIG. 6. (Color online) $v_{4} /\left(v_{2}\right)^{2}$ for charged hadrons calculated taking or not taking into account the chiral fields as sources for the hydrodynamic equations, for $g=3,3.2,3.4$ and a temperaturedependent $\eta / s$.

As with $v_{2}$, it is important to determine the dependence of our results and conclusions on the value of $g$. Figure 6 shows $v_{4} /\left(v_{2}\right)^{2}$ calculated including or not the sources in the hydrodynamic equations, for $g=3,3.2,3.4$ and a temperature-dependent $\eta / s$. It is seen that the difference in the values of $v_{4} /\left(v_{2}\right)^{2}$ calculated including or not the chiral fields as sources becomes smaller with increasing $g$, but is still relatively large at $g=3.4$. Moreover, it is seen that the behavior of this ratio with $p_{T}$ does not depend strongly on the value of $g$. These results obtained with different values of $g$ support the conclusion mentioned before that $v_{4} /\left(v_{2}\right)^{2}$ is more sensitive than $v_{2}$ to the influence of the chiral fields on the evolution of the quark fluid.

\section{CONCLUSIONS}

We have studied the evolution of the fireball created at RHIC within second-order viscous hydrodynamics coupled self-consistently to the LSM, focusing on the impact of the dynamics of the chiral fields on the hadronic observables $v_{2}$ and $v_{4} /\left(v_{2}\right)^{2}$. We have compared the results obtained when the chiral fields are included or not as sources in the hydrodynamic equations. The comparisons were made using both temperature-independent and temperature-dependent $\eta / s$. The temperature dependence of $\eta / s$ was calculated in the LSM using the linearized Boltzmann equation.

We have found that the values of $v_{2}$ do not depend strongly on the evolution of the chiral fields. Specifically, for a temperature-independent $\eta / s$ this dependence is negligible for $p_{T}<3 \mathrm{GeV}$, while for a temperature-dependent $\eta / s$ it is appreciable but still small even at small $p_{T}$. We have also found that the ratio $v_{4} /\left(v_{2}\right)^{2}$ is much more sensitive to the dynamics of the chiral fields, being larger when these fields are taken into account as sources in the hydrodynamic equations, in both situations of the temperature dependence of $\eta / s$.

In line with the results of Refs. [8-10], our results show that not knowing precisely the temperature dependence of $\eta / s$ leads to further uncertainties in attempts of extracting this ratio from data on $v_{2}$, in addition to the uncertainties that stem from the initial conditions and the freeze-out process, among others sources. It is worth noting that despite the coupling of chiral sources to the hydrodynamic evolution would add further uncertainties, they are not very big.

The model used in this work leaves room for improvements in different directions. Probably, the most important ones for our analysis are including bulk viscosity in the hydrodynamic equations and fluctuations of the chiral fields. This latter effect would act as noise sources in the classical equations of motion. We believe that the qualitative trends and the general conclusions extracted from our results will hold when these effects are taken into account. Work is in progress where these two aspects are taken into account in a viscous hydrodynamic simulation and their impact on observables will be reported in a forthcoming publication.

\section{ACKNOWLEDGMENTS}

We thank Jorge Noronha for useful comments on the calculation of transport coefficients of strongly coupled theories. This work was partially funded by Conselho Nacional de Desenvolvimento Científico e Tecnológico (Brazil's National Council of Technological and Scientific Development) and Fundação de Amparo à Pesquisa do Estado de São Paulo (Foundation for Research Support of the State of São Paulo).

\section{APPENDIX A: LINEAR $\sigma$ MODEL}

In this Appendix we will briefly review the relevant aspects of the linear $\sigma$ model for the present paper, as well as the calculation of $\eta$ from the linearized Boltzmann equation in the relaxation time approximation.

As mentioned before, as an effective theory of the chiral symmetry-breaking dynamics we consider the linear $\sigma$ model coupled to two flavors of constitutive quarks. The Lagrangian density of the coupled system is given in Eq. (1). The quark fluid is considered as a thermal bath for the chiral field, and therefore it can be integrated out to obtain the effective potential $V_{e}$ for the chiral fields in the presence of that bath of quarks. To calculate the equilibrium pressure density $p(\phi, T)=-V_{e}(\phi, T)+U(\phi)$, we use the one-loop effective potential $[41,42]$ :

$$
V_{e}(\phi, T)=U(\phi)-d_{q} T \int \frac{d^{3} p}{(2 \pi)^{3}} \ln \left(1+e^{-E / T}\right),
$$

where $d_{q}=24$ is the color-spin-isospin-baryon charge degeneracy of the quarks and the energy $E=\left(p^{2}+m_{q}^{2}\right)^{1 / 2}$ with $m_{q}^{2}=g^{2} \phi^{2}$. The equilibrium energy density is just

$$
\epsilon(\phi, T)=d_{q} \int \frac{d^{3} p}{(2 \pi)^{3}} f_{0} E .
$$

From $p$ and $\epsilon$, the square of the speed of sound and the entropy density follow: $c_{s}^{2}=d p / d \epsilon$ and $s=\partial p / \partial T$. 
The local thermal averages of the scalar and pseudovector chiral densities $\rho_{s}=\langle\bar{q} q\rangle$ and $\vec{\rho}_{p s}=\left\langle\bar{q} \gamma_{5} \vec{\tau} q\right\rangle$ enter as sources in the hydrodynamic equations. Explicitly, they are given by

$$
\begin{aligned}
\rho_{s} & =g \sigma d_{q} \int \frac{d^{3} p}{(2 \pi)^{3}} \frac{1}{E} f_{0}, \\
\vec{\rho}_{p s} & =g \vec{\pi} d_{q} \int \frac{d^{3} p}{(2 \pi)^{3}} \frac{1}{E} f_{0} .
\end{aligned}
$$

The LSM exhibits a first-order phase transition, a crossover, and a critical end point, depending on the value of the chiral coupling constant $g$. The crossover phase transition is not a genuine phase transition because all thermodynamic functions change smoothly with temperature. However, such changes may be quite sudden in a narrow temperature range. As indicated in Sec. IV, throughout this work we set $g=3, g=$ 3.2 , and $g=3.4$, which correspond to a smooth crossover and lead to a temperature-dependent sound speed that resembles that of lattice QCD.

Here we model the expansion of the QGP using viscous hydrodynamics with the bulk viscosity $\zeta$ set to zero. It is known that $\zeta$ scales as $c_{s}^{2}-1 / 3$, so from Fig. 1 it is clear that for the LSM $\zeta \neq 0$ (see also Refs. [28,29]). Recent lattice QCD results indicate that the quark-gluon matter EOS departs from the conformal description [70-74], although it is common practice to neglect $\zeta$ in the hydrodynamic equations while using an EOS inspired on lattice QCD. An estimate for the temperature dependence and the relative importance of $\zeta$ can be obtained from $(\epsilon-3 p) / T^{4}$, which measures the deviation from the conformal limit. Figure 7 shows this quantity calculated in the LSM as a function of temperature, for $g=3$, 3.2, 3.4. It is seen that, for the range of temperatures of interest $T \geqslant 130 \mathrm{MeV},(\epsilon-3 p) / T^{4}$ is small except at $T \leqslant T_{c}$ where it rises abruptly reaching significant values. The increase in $(\epsilon-3 p) / T^{4}$ near $T_{c}$ is sharper for larger values of $g$. It was shown before that the hydrodynamic evolution is affected by bulk viscosity, for example, reducing the elliptic flow, inducing the phenomenon of cavitation or modifying the freeze-out process $[13,79,80]$. Although it is reasonable to assume that these changes will not affect in a dramatic way

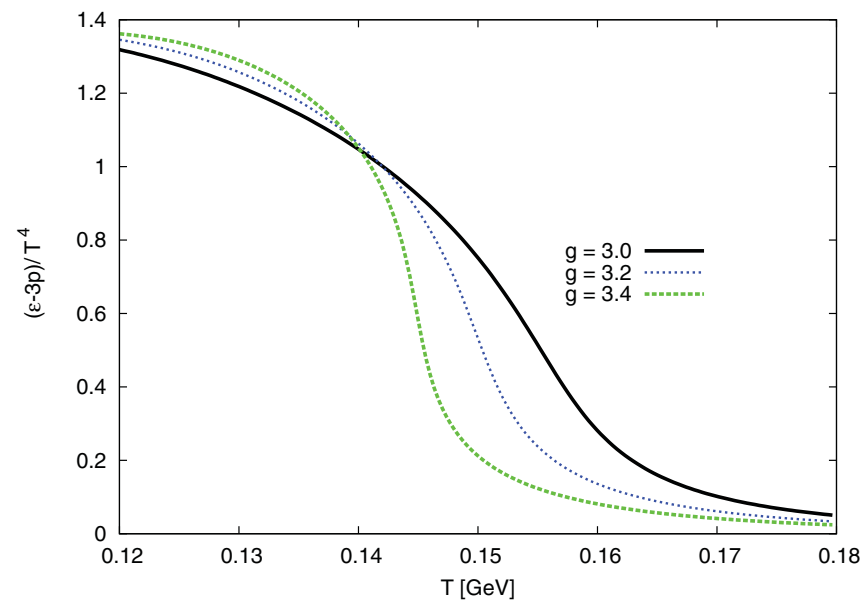

FIG. 7. (Color online) $(\epsilon-3 p) / T^{4}$ of the LSM as a function of temperature for $g=3,3.2,3.4$. the differences in $v_{2}$ and $v_{4} /\left(v_{2}\right)^{2}$ that result from including or not the chiral fields as sources for the evolution of the quark fluid, a more thorough investigation of this particular issue is necessary.

We now go over to the calculation of the shear viscosity. To calculate $\eta$ we adopt the results of Ref. [28], which are based on the linearized Boltzmann equation in the relaxation time approximation. In the relaxation time approximation the shear viscosity is given by

$$
\eta=\frac{4 \tau}{5 T} \int \frac{d^{3} p}{(2 \pi)^{3}} \frac{p^{4}}{E^{2}} f_{0}\left(1-f_{0}\right),
$$

where $\tau=\tau(T)$ is the collision time. $\tau(T)$ is calculated from the averaged cross sections $\bar{\sigma}$ for quark-quark and quark-antiquark scattering processes including $1 / N_{c}$ next to leading order corrections as

$\tau^{-1}=6 f_{0}\left(\bar{\sigma}_{u u \rightarrow u u}+\bar{\sigma}_{u d \rightarrow u d}+\bar{\sigma}_{u \bar{u} \rightarrow u \bar{u}}+\bar{\sigma}_{u \bar{u} \rightarrow d \bar{d}}+\bar{\sigma}_{u \bar{d} \rightarrow u \bar{d}}\right)$.

We refer the reader to Refs. [28,94,95] for details on the calculation of the $\bar{\sigma}^{\prime} s$; we note that the chiral model used in these references is not very different from the LSM, and the exchanges of $\sigma$ and $\pi$ mesons are modeled by contact terms. Note also that the cross sections are temperature dependent, not only because of phase space, but also because they depend on the constituent quark masses, whose temperature dependence is given by the LSM of the present paper. See also Ref. [29] for a related approach applied to the calculation of $\eta / s$ in the LSM of a pion gas.

\section{APPENDIX B: DEPENDENCE OF RESULTS ON CUTOFF FOR $\eta / s$}

It was noted before that any hydrodynamical description of matter is bound to become inapplicable at large viscosity. Because our simulations are purely hydrodynamic and the

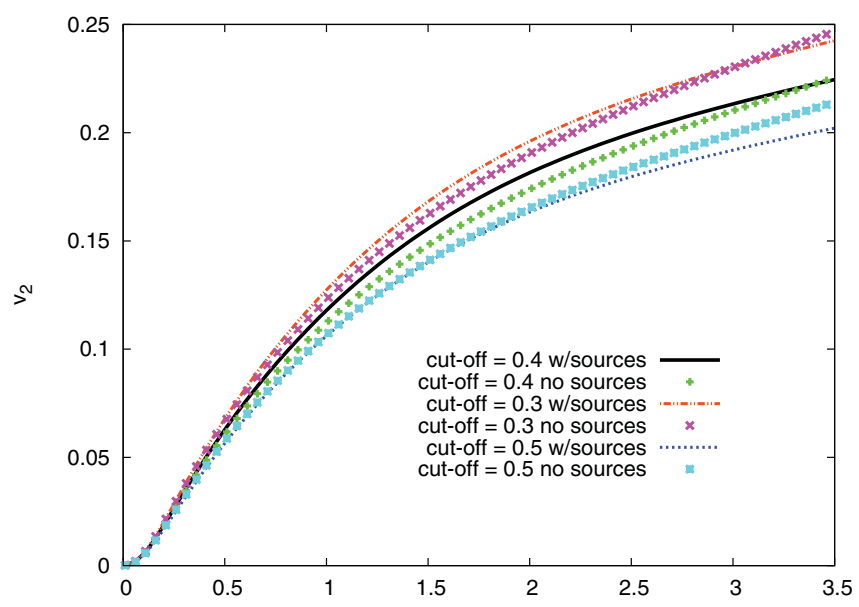

FIG. 8. (Color online) $v_{2}$ for charged hadrons calculated taking or not taking into account the chiral fields as sources for the hydrodynamic equations calculated with different choices for the cutoff value $\eta / s=0.3,0.4,0.5$. The value of the coupling constant is $g=3.2$. 


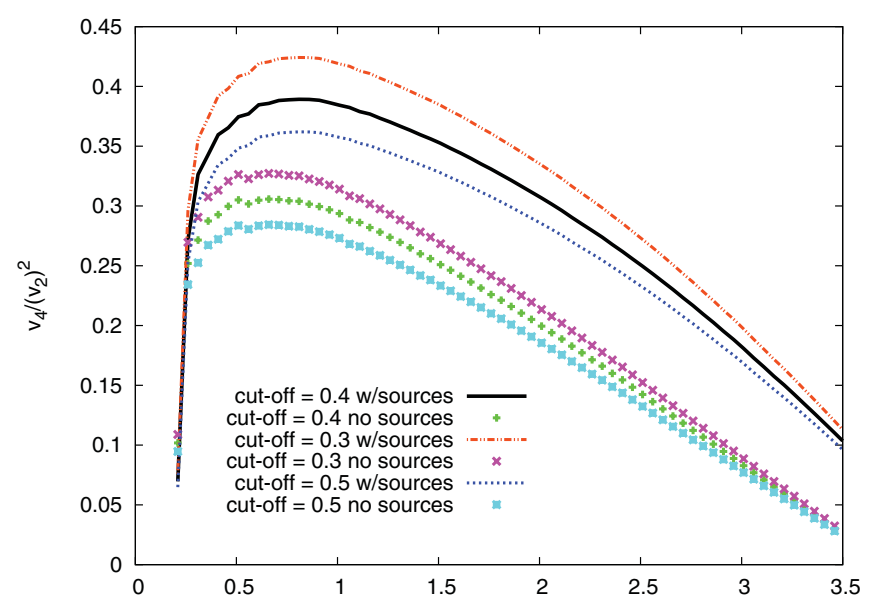

FIG. 9. (Color online) $v_{4} /\left(v_{2}\right)^{2}$ for charged hadrons calculated taking or not taking into account the chiral fields as sources for the hydrodynamic equations calculated with different choices for the cutoff value $\eta / s=0.3,0.4,0.5$. The value of the coupling constant is $g=3.2$.

calculated $\eta / s$ increases rapidly with decreasing temperature, a cutoff for the value of $\eta / s$ must be imposed. The comparison of the results obtained from hydrodynamic and kinetic simulations to data provide a guide as to what is the value at which one should impose the cutoff. In our simulations we set the cutoff at $\eta / s=0.4$, which is in the range of values found to correspond to the breakdown of viscous hydrodynamics
[50,77]. In this Appendix we show the results for hadronic observables calculated using different values of this cut-off, namely $\eta / s \leqslant 0.3,0.4$, and 0.5 , and analyze the dependence of the results on this choice for the cut-off. For simplicity, the results shown in this Appendix correspond to $g=3.2$.

Figures 8 and 9 show the charged-hadron $v_{2}$ and $v_{4} /\left(v_{2}\right)^{2}$, respectively, as a function of transverse momentum, obtained with cutoff values of $\eta / s=0.3,0.4$, and 0.5 . It is seen that although the values of $v_{2}$ and $v_{4} /\left(v_{2}\right)^{2}$ change with the value of the cutoff, the relation between the results obtained including or not the chiral fields as sources in the hydrodynamic equations remains practically the same. Consequently, the conclusions extracted from these results, which are discussed in the main text, do not depend on the precise value of the cutoff imposed on $\eta / s$, provided that $g \sim 3.2$ corresponding to a smooth crossover.

As a final side remark, it is interesting to note that if one would attempt to extract the value of $\eta / s$ by matching $v_{2}$ to data (with the remark mentioned in Sec. I), the uncertainty in the extracted $\eta / s$ coming from the choice of cutoff in the range $[0.3,0.5]$ would be $\sim 50 \%$ with respect to the average value of $\eta / s$. This relatively large value of the uncertainty in $\eta / s$ associated with the imposed cutoff clearly displays the sensitivity of observables on the shear viscosity of the hadronic stage in collisions at $\sqrt{s_{N N}}=200 \mathrm{GeV}$, a fact already discussed in Refs. [8-11], pointing to the conclusion that further study is needed to extract the precise temperature dependence of $\eta / s$ from heavy-ion collision experiments.
[1] S. S. Adler et al. (PHENIX Collaboration), Phys. Rev. Lett. 91, 182301 (2003).

[2] J. Adams et al. (STAR Collaboration), Phys. Rev. Lett. 92, 112301 (2004).

[3] K. Adcox et al. (PHENIX Collaboration), Phys. Rev. C 69, 024904 (2004).

[4] J. Adams et al. (STAR Collaboration), Phys. Rev. C 72, 014904 (2005).

[5] I. Arsene et al. (BRAHMS Collaboration), Phys. Rev. C 72, 014908 (2005).

[6] B. B. Back et al. (PHOBOS Collaboration), Phys. Rev. C 72, 051901(R) (2005).

[7] J.-Y. Ollitrault, Phys. Rev. D 46, 229 (1992).

[8] H. Niemi, G. S. Denicol, P. Huovinen, E. Molnar, and D. H. Rischke, Phys. Rev. Lett. 106, 212302 (2011).

[9] J. L. Nagle, I. G. Bearden, and W. A. Zajc, arXiv:1102.0680 [nucl-th].

[10] C. Shen and U. W. Heinz, Phys. Rev. C 83, 044909 (2011).

[11] P. Bozek, Phys. Rev. C 81, 034909 (2010).

[12] G. S. Denicol, T. Kodama, and T. Koide, arXiv:1002.2394v1 [nucl-th].

[13] J. R. Bhatt, H. Mishra, and V. Sreekanth, J. High Energy Phys. 11 (2010) 106.

[14] H. Song and U. W. Heinz, Phys. Rev. C 81, 024905 (2010).

[15] K. Kanaya, PoS LATTICE2010, 012 (2010).

[16] A. Nakamura and S. Sakai, Phys. Rev. Lett. 94, 072305 (2005).

[17] H. B. Meyer, Phys. Rev. D 76, 101701 (2007).
[18] H. B. Meyer, Phys. Rev. Lett. 100, 162001 (2008).

[19] P. B. Arnold, G. D. Moore, and L. G. Yaffe, J. High Energy Phys. 11 (2000) 001; 05 (2003) 051.

[20] P. B. Arnold, C. Dogan, and G. D. Moore, Phys. Rev. D 74, 085021 (2006).

[21] M. Prakash, M. Prakash, R. Venugopalan, and G. Welke, Phys. Rep. 227, 321 (1993).

[22] J. W. Chen and J. Wang, Phys. Rev. C 79, 044913 (2009).

[23] F. Wilczek, arXiv:hep-ph/0003183.

[24] A. Dobado and F. J. Llanes-Estrada, Phys. Rev. D 69, 116004 (2004).

[25] A. Muronga, Phys. Rev. C 69, 044901 (2004).

[26] J. W. Chen, Y. H. Li, Y. F. Liu, and E. Nakano, Phys. Rev. D 76, 114011 (2007).

[27] K. Itakura, O. Morimatsu, and H. Otomo, Phys. Rev. D 77, 014014 (2008).

[28] C. Sasaki and K. Redlich, Phys. Rev. C 79, 055207 (2009); Nucl. Phys. A 832, 62 (2010).

[29] P. Chakraborty and J. I. Kapusta, Phys. Rev. C 83, 014906 (2011).

[30] J. Noronha-Hostler, Jorge Noronha, and C. Greiner, Phys. Rev. Lett. 103, 172302 (2009).

[31] L. P. Csernai, J. I. Kapusta, and L. D. McLerran, Phys. Rev. Lett. 97, 152303 (2006).

[32] R. Baier, P. Romatschke, D. T. Son, A. O. Starinets, and M. A. Stephanov, J. High Energy Phys. 04 (2008) 100.

[33] S. Bhattacharyya, V. E. Ehubeny, S. Minwalla, and M. Rangamani, J. High Energy Phys. 02 (2008) 045. 
[34] M. Natsuume and T. Okamura, Phys. Rev. D 77, 066014 (2008); 78, 089902(E) (2008).

[35] M. Gell-Mann and M. Levy, Nuovo Cim. 16, 705 (1960).

[36] I. N. Mishustin and O. Scavenius, Phys. Rev. Lett. 83, 3134 (1999).

[37] A. Abada and M. C. Birse, Phys. Rev. D 55, 6887 (1997).

[38] D. T. Son, Phys. Rev. Lett. 84, 3771 (2000).

[39] C. Pujol and D. Davesne, Phys. Rev. C 67, 014901 (2003).

[40] Y. Lallouet, D. Davesne, and C. Pujol, Phys. Rev. C 67, 057901 (2003).

[41] K. Paech, H. Stöcker, and A. Dumitru, Phys. Rev. C 68, 044907 (2003).

[42] K. Paech and A. Dumitru, Phys. Lett. B 623, 200 (2005).

[43] M. Nahrgang and M. Bleicher, J. Phys. Conf. Ser. 270, 012059 (2010).

[44] M. Nahrgang, S. Leupold, C. Herold, and M. Bleicher, Phys. Rev. C 84, 024912 (2011).

[45] M. Nahrgang, S. Leupold, and M. Bleicher, arXiv:1105.1396 [nucl-th].

[46] M. Nahrgang, M. Bleicher, S. Leupold, and I. Mishustin, arXiv:1105.1962 [nucl-th].

[47] S. Plumari, V. Baran, M. Di Toro, G. Ferini, and V. Greco, Phys. Lett. B 689, 18 (2010).

[48] M. Luzum and P. Romatschke, Phys. Rev. C 78, 034915 (2008); 79, 039903 (2009).

[49] U. W. Heinz, arXiv:0901.4355 [nucl-th].

[50] P. Romatschke, Int. J. Mod. Phys. E 19, 1 (2010)

[51] J. Peralta-Ramos and E. Calzetta, Phys. Rev. C 82, 054905 (2010).

[52] R. P. G. Andrade, F. Grassi, Y. Hama, T. Kodama, and W. L. Qian, Phys. Rev. Lett. 101, 112301 (2008).

[53] H. Petersen, C. Coleman-Smith, S. A. Bass, and R. Wolpert, J. Phys. G 38, 045102 (2011).

[54] R. S. Bhalerao, M. Luzum, and J.-Y. Ollitrault, arXiv:1104.4740v1 [nucl-th].

[55] W. Israel, Ann. Phys. (NY) 100, 310 (1976).

[56] W. Israel and J. M. Stewart, Phys. Lett. A 58, 213 (1976).

[57] W. Israel and J. M. Stewart, Ann. Phys. (NY) 118, 341 (1979).

[58] B. Betz, D. Henkel, and D. H. Rischke, J. Phys. G 36, 064029 (2009).

[59] J. Noronha, and G. S. Denicol, arXiv:1104.2415v2 [hep-th].

[60] G. S. Denicol, J. Noronha, H. Niemi, and D. H. Rischke, Phys. Rev. D 83, 074019 (2011).

[61] T. Koide and T. Kodama, Phys. Rev. E 78, 051107 (2008).

[62] F. Cooper and G. Frye, Phys. Rev. D 10, 186 (1974).

[63] S. R. de Groot, W. A. van Leeuwen and Ch. G. van Weert, Relativistic Kinetic Theory (North-Holland, Netherlands, 1980).

[64] P. F. Kolb, J. Sollfrank, and U. Heinz, Phys. Rev. C 62, 054909 (2000).
[65] P. F. Kolb and R. Rapp, Phys. Rev. C 67, 044903 (2003).

[66] P. F. Kolb and U. Heinz, arXiv:nucl-th/0305084. AZHYDRO code v0.2 is available from [http://karman.physics.purdue.edu/OSCAR].

[67] W. Florkowski, Nucl. Phys. A 853, 173 (2011).

[68] M. Chojnacki and W. Florkowski, Acta Phys. Polon. B 38, 3249 (2007).

[69] S. Pratt, Phys. Rev. Lett. 102, 232301 (2009).

[70] Y. Aoki, G. Endrodi, Z. Fodor, S. D. Katz, and K. K. Szabo, Nature (London) 443, 675 (2006).

[71] S. Borsanyi, Z. Fodor, C. Hoelbling, S. D. Katz, S. Krieg, C. Ratti, and K. K. Szabo, arXiv:1005.3508v1 [hep-lat].

[72] R. A. Soltz (for the HotQCD Collaboration), Nucl. Phys. A 830, 725 (2009).

[73] M. Cheng et al., Phys. Rev. D 74, 054507 (2006).

[74] M. Laine and Y. Schroder, Phys. Rev. D 73, 085009 (2006).

[75] H. Song, S. A. Bass, and U. W. Heinz, Phys. Rev. C 83, 024912 (2011).

[76] D. Fernandez-Fraile and A. Gomez Nicola, arXiv:0912.4002v1 [hep-ph].

[77] D. A. Teaney, arXiv:0905.2433v1 [nucl-th].

[78] S. Pratt and G. Torrieri, Phys. Rev. C 82, 044901 (2010).

[79] H. Song and U. W. Heinz, Phys. Rev. C 81, 024905 (2010).

[80] A. Monnai and T. Hirano, Phys. Rev. C 80, 054906 (2009).

[81] P. Huovinen and D. Molnar, Phys. Rev. C 79, 014906 (2009).

[82] K. Aamodt et al. (ALICE Collaboration), Phys. Rev. Lett. 107, 032301 (2011).

[83] C. Shen, S. A. Bass, T. Hirano, P. Huovinen, Z. Qiu, H. Song, and U. W. Heinz, arXiv:1106.6350v1 [nucl-th].

[84] B. Schenke, arXiv:1106.6012v1 [nucl-th].

[85] J. L. Nagle and M. P. McCumber, Phys. Rev. C 83, 044908 (2011).

[86] R. A. Lacey, R. Wei, J. Jia, N. N. Ajitanand, J. M. Alexander, and A. Taranenko, Phys. Rev. C 83, 044902 (2011).

[87] R. A. Lacey, R. Wei, N. N. Ajitanand, J. M. Alexander, X. Gong, J. Jia, A. Taranenko, R. Pak, and H. Stöcker, Phys. Rev. C 81, 061901 (2010).

[88] M. Luzum, C. Gombeaud, and J.-Y. Ollitrault, Phys. Rev. C 81, 054910 (2010).

[89] M. Luzum and J.-Y. Ollitrault, Phys. Rev. C 82, 014906 (2010).

[90] E. Calzetta and J. Peralta-Ramos, Phys. Rev. D 82, 106003 (2010).

[91] K. Dusling, G. D. Moore, and D. Teaney, Phys. Rev. C 81, 034907 (2010).

[92] A. Monnai and T. Hirano, Phys. Rev. C 80, 054906 (2009).

[93] G. S. Denicol, T. Kodama, T. Koide, and Ph. Mota, Phys. Rev. C 80, 064901 (2009).

[94] P. Zhuang, J. Hufner, S. P. Klevansky, and L. Neise, Phys. Rev. D 51, 3728 (1995).

[95] P. Rehberg, S. P. Klevansky, and J. Hufner, Nucl. Phys. A 608, 356 (1996). 